NOTA CIENTÍFICA

\title{
Bioensayos preliminares para evaluar la toxicidad del lindano sobre Simocephalus vetulus (O.F.Muller, 1776) (Crustacea: Cladocera)
}

\section{Preliminary bioassays to evaluate the toxicity of lindano on Simocephalus vetulus (O.F.Muller, 1776) (Crustacea: Cladocera)}

\author{
Jorgelina Juárez* y Alcira Villagra de Gamundi
}

*Instituto de Limnología (ILINOA), Facultad de Ciencias Naturales e Instituto Miguel Lillo, Universidad Nacional De Tucuman, Argentina.

E-mail Jorgelina Juárez: jorgelina_j@yahoo.com

Presentado: $\quad 20 / 12 / 2006$ Aceptado: $\quad 18 / 05 / 2007$

\section{Resumen:}

El lindano es conocido por su toxicidad y su capacidad de bioacumulación en los seres vivos, convirtiéndose en un compuesto peligroso para la salud humana y el ambiente. El objetivo del presente trabajo fue evaluar la toxicidad aguda del lindano sobre el cladócero Simocephalus vetulus. Se realizaron cultivos en cámara de incubación en condiciones controladas con medios diferenciales (algas e infusorios). Los bioensayos se implementaron con un control, 5 diluciones y 3 réplicas. El valor de CL50 a 48 horas de exposición frente a las distintas concentraciones de lindano fue de $11.4 \mu \mathrm{g} / \mathrm{l}$, lo que refleja la mayor sensibilidad del organismo prueba en relación a otros antecedentes.

Palabras claves: Bioensayos, toxicidad, lindano, Simocephalus vetulus

\section{Abstract}

Lindano is known by its toxicity and its capacity of bioacumulation in organisms, becoming a material dangerous for the human health and the environment. The objective of the present work was to evaluate the acute toxicity of the lindane on the cladoceran Simocephalus vetulus. Cultures were made in camera of incubation in conditions controlled with average differentials (seaweed and infusorians). The bioensayos were implemented with a control, 5 dilutions and 3 retorts. The value of CL50 to 48 hours of exhibition as opposed to the different concentrations from lindane was of 11,4 $\mu \mathrm{g} / \mathrm{l}$, which reflects the greater sensitivity of the organism proves in relation to other antecedents.

Keywords: Bioensayos, toxicity, lindane, Simocephalus vetulus

\section{Introducción}

Tucumán, en Argentina, sufre una alarmante situación frente a las sustancias tóxicas. El mal manejo de los plaguicidas, la falta de tratamiento adecuado de los efluentes industriales y urbanos, el no-cumplimiento de la legislación vigente, entre otros, son problemas que convergen para determinar una alta complejidad en su tratamiento (Sosa et al., 1999). El lindano es un insecticida organoclorado, relativamente volátil y persistente categorizado como moderadamente peligroso (clase II) (CASAFE, 1999). Por su estabilidad química y su gran afinidad con las grasas, se bioacumula a través de la cadena trófica, detectándose su presencia en tejido adiposo de animales, y hombre (riñón, hígado, leche materna y suero sanguíneo humano). Puede migrar largas distancias a través del aire en forma de vapores o adherido a partículas distribuyéndose en todo el mundo. Es usado en múltiples actividades productivas y domésticas (como insecticida en el control de plagas agropecuarias, en salud pública o en aplicaciones farmacéuticas, especialmente como piojicidas). La contaminación aguda o crónica con lindano tiene varios efectos en la salud humana, afectando básicamente al sistema nervioso central, tracto digestivo (vómitos, convulsiones, parálisis, hemorragia y coma) como también al sistema endocrino con efecto cancerígeno. Debido a ello esta sustancia está prohibida en numerosos países como Australia, Austria, Indonesia, Nueva Zelanda, Holanda, Canadá y EE.UU. (Instituto Nacional de Ecología, 2004), en consideración del riesgo ecológico y efectos adversos en la fauna terrestre y acuática que pueda causar (Iannacone et al., 2000). En Argentina el lindano esta restringido para distintos usos: a)
En Sanidad Animal (Decreto 2143/68) y más tarde se prohibió completamente su uso veterinario (Resolución 513/98), b) En Sanidad Vegetal (Disp. 80/71), después se lo prohibió como gorgojicida (Disp. 47/72) y finalmente se prohibió completamente su uso en vegetales (Resolución 513/98) y c) Como Insecticida Domisanitario (Resolución 7292/98) (Digón et al., 2005). Por esta razón es importante determinar el nivel de riego ambiental del plaguicida lindano sobre organismos representantes del ecosistema acuático utilizando bioensayos de toxicidad. En el presente trabajo se seleccionó como organismo prueba al cladócero Simocephalus vetulus especie siempre asociada a la vegetación sumergida y presente en pequeños cuerpos de agua; estancados o corrientes, a veces con marcada contaminación. Se alimenta de algas, protozoos y detritos orgánicos. Los juveniles alcanzan la madurez sexual alrededor de los 9 días.

El objetivo del presente trabajo fue evaluar la toxicidad aguda del lindano sobre el cladócero Simocephalus vetulus.

Condiciones de laboratorio: Simocephalus vetulus fue colectado en un estanque artificial ubicado en la Facultad de Ciencias Naturales e Instituto Miguel Lillo (Universidad Nacional de Tucumán, Argentina) y se cultivaron en el laboratorio del Instituto de Limnología del Noroeste Argentino perteneciente a la misma institución. Se usaron recipientes de vidrio de 1,5 litros de capacidad con 1 litro de medio natural filtrado, proveniente del estanque original. Se mantuvieron durante 30 días en fase de aclimatación, en una cámara de incubación Biological - Sca 
Tabla 1. Condiciones de prueba de toxicidad aguda

\begin{tabular}{|c|c|}
\hline Factor & Condición \\
\hline Temperatura & $25 \pm 2{ }^{\circ} \mathrm{C}$ \\
\hline Fotoperíodo & 12:12 h Luz/oscuridad \\
\hline Envase prueba & $20 \mathrm{ml}$ \\
\hline Volumen de exposición & $10 \mathrm{ml}$ \\
\hline $\begin{array}{l}\text { Edad de organismos } \\
\text { prueba }\end{array}$ & Neonatos $24 \mathrm{~h}$ \\
\hline $\begin{array}{l}\text { No de réplicas por } \\
\text { concentración }\end{array}$ & 3 \\
\hline $\begin{array}{l}\mathrm{N}^{\mathrm{o}} \text { de concentraciones } \\
\text { más control }\end{array}$ & $6(1,2 ; 2,8 ; 7,2 ; 18$ y $45 \mu \mathrm{g} / 1)$ \\
\hline $\begin{array}{l}\mathrm{N}^{\circ} \text { de organismos } \\
\text { por envase }\end{array}$ & 10 \\
\hline $\begin{array}{l}\mathrm{N}^{\mathrm{o}} \text { de organismos totales } \\
\text { por concentración }\end{array}$ & 30 \\
\hline Alimentación & No requiere \\
\hline Agua de dilución & Agua natural filtrada \\
\hline Tiempo de exposición & $48 \mathrm{~h}$ \\
\hline Respuesta letal & Mortalidad, sin movilidad \\
\hline
\end{tabular}

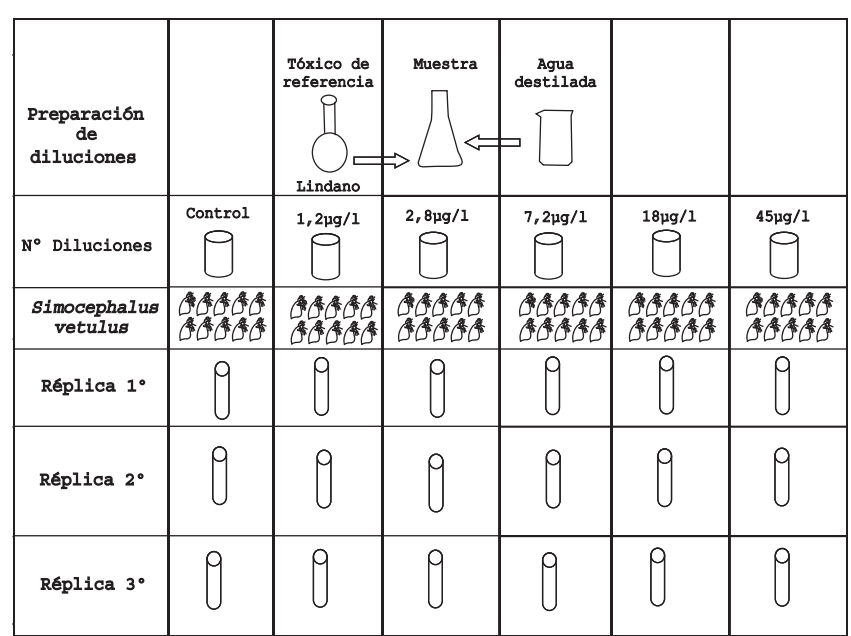

Figura 1. Esquema del diseño experimental para evaluar la toxicidad del Lindano sobre Simocephalus vetulus.

1000 en condiciones controladas con un fotoperíodo de 12:12 $\mathrm{h}$ luz/oscuridad y temperatura $=25 \pm 2{ }^{\circ} \mathrm{C}$ (Tabla 1$)$. Se alimentaron 2 veces por semana con cultivo monoespecífico del alga Selenastrun capricornutum (ISO, 1989; Espinosa-Chávez et al., 1992) ( $1 \mathrm{ml}$ de concentrado/litro de medio) y con infusorios (1 g/litro de medio).

Diseño de experimento: Para la realización de los bioensayos, a partir de una solución stock de lindano marca Rydel-de Haëden, obtenido del Instituto de Evaluación y Control de contaminantes Ambientales (LECCA) de la Facultad de Ciencias Exactas y Tecnología de la Universidad Nacional de Tucumán, se prepararon 5 diluciones y un control. En recipientes de $20 \mathrm{ml}$ se colocaron $10 \mathrm{ml}$ de la dilución correspondiente y 10 neonatos

Tabla 2. Porcentaje de Mortalidad de Simocephalus vetulus en experimento para evaluar toxicidad del Lindano.

\begin{tabular}{ccccccc}
\hline $\begin{array}{l}\mathbf{N}^{\mathbf{0}} \text { de } \\
\text { réplica }\end{array}$ & Control & $\mathbf{1 , 2}$ & $\begin{array}{c}\mathbf{m g} / \mathbf{1} \\
\mathbf{2 , 8}\end{array}$ & $\mathbf{7 , 2}$ & $\mathbf{1 8}$ & $\mathbf{4 5}$ \\
\hline 1 & 93 & 90 & 86 & 83 & 100 & 100 \\
2 & 26 & 40 & 73 & 80 & 100 & 100 \\
3 & 23 & 53 & 63 & 83 & 96 & 100 \\
Total & 47,3 & 61 & 74 & 82 & 98,6 & 100 \\
\hline
\end{tabular}

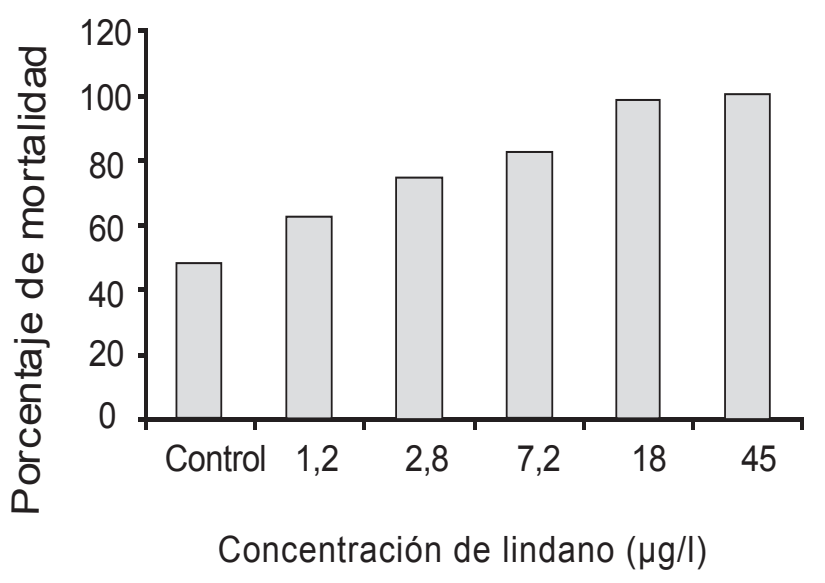

Figura 2. Promedio del porcentaje de mortalidad de Simocephalus vetulus, causada por diferentes concentraciones de Lindano.

de aproximadamente $24 \mathrm{~h}$ de edad. Se realizaron 3 réplicas por concentración (Fig. 1). El tiempo total de la prueba fue de $48 \mathrm{~h}$ manteniendo los recipientes bajo las mismas condiciones de cultivo. Posteriormente se realizó el recuento de individuos muertos (inmóviles) por concentración, determinándose el porcentaje de mortalidad para cada una (Silva et al., 2003; ISO, 1982).

Para expresar los resultados obtenidos se elaboraron gráficas de dosis-respuesta y se calculó la concentración que produce el $50 \%$ de mortalidad, mediante el programa estadístico Probit (US EPA, 1988).

\section{Resultados}

El porcentaje de mortalidad de neonatos en la batería experimental fue el mínimo en el control (47.3\%) y máximo (100\%) a partir de $18 \mu \mathrm{g} / \mathrm{l}$ (Tabla 2 y Figura 2).

El valor de CL50 a 48 horas de exposición frente a las distintas concentraciones de lindano $(1,2 ; 2,8 ; 7,2 ; 18$ y $45 \mu \mathrm{g} / \mathrm{l})$ fue de 11,4 $\mu \mathrm{g} / \mathrm{l}$ (Tabla 3).

\section{Discusión y conclusiones}

El cladócero Simocephalus vetulus posee un tamaño apropiado (3 a $4 \mathrm{~mm}$ en especimenes adultos) que permite visualizarlo y manipularlo con mayor facilidad. Además permiten disponer de abundantes especimenes idénticos en un tiempo relativamente reducido ya que las hembras se reproducen partenogenéticamente y poseen un corto tiempo generacional (8-12 días en condiciones óptimas) (Díaz Báez et al., 2004), condiciones óptimas para su cultivo y manipulación en laboratorio. Además este taxón se registra frecuentemente en humedales regionales y locales lo que favorece su uso como organismo prueba.

Con los presentes resultados confirmamos la alta susceptibilidad de $S$. vetulus a tóxicos. Existen antecedentes que demuestran un CL50 hasta 30,8 veces mayor para el cloruro de mercurio y de menor grado para el acetato de etilo $(1,27)$ y cadmio $(5,71)$ comparado con D. magna, como también de 4,46 para el titanio

Tabla 3. Valores de CL/CE estimados y Límites de Confianza $(95 \%)$ proporcionados por el programa Probit $(\mu \mathrm{g} / \mathrm{l})$

\begin{tabular}{lccc}
\hline Puntos & Concentración & Inferior & Superior \\
\hline LC/CE 1.0 & 1,253 & 0,011 & 4,238 \\
LC/CE 50.0 & 11,413 & 2,540 & 20,215 \\
\hline
\end{tabular}


frente a D. pulex. (Rico Martínez, 2000). En tal sentido resultó también ser más sensible al lindano $(11,4 \mathrm{mg} / \mathrm{l})$ con un valor de CL50 40 veces mayor respecto a $460 \mathrm{mg} / \mathrm{l}$ para Daphnia (Silva et al., 2003) lo que le otorga una valoración interesante potenciándolo como biosensor de contaminantes.

\section{Literatura citada}

CASAFE. 1999. Guía de productos fitosanitarios para la República Argentina. 1600 p. 9ª ed. Ed. Cámara de Sanidad Agropecuaria y Fertilizantes, Buenos Aires, Argentina.

Díaz Báez, M. C., Y. Pica Granados \& A. Ronco. 2004. Bioensayo de toxicidad aguda con Daphnia magna. Ensayos Ecotoxicológicos y Métodos de Evaluación de calidad de Aguas. Estandarización, intercalibración, resultados y aplicaciones. Centro Internacional de investigaciones para el desarrollo. Instituto Mexicano de Tecnología del Agua (IMTA). 4. (2): Pp. 4.

Digón A. \& A. Giorgio. 2005. Sustancias o compuestos químicos prohibidos o restringidos en la República Argentina. Programa Nacional de Riesgos Químicos - Plan nacional De Gestión de Sustancias Químicas. Ministerio de salud (Dirección de Promoción y Protección de la Salud); Ministerio de Defensa (Dirección General de Planificación Tecnológica e Industrial). Secretaría de agricultura, Ganadería, Pesca y Alimentación (Coordinación de Registro de Agroquímicos). $<\mathrm{http}: / / \mathrm{msal}$.gov.ar/htm/site/pdf/restringida s\%20y\%20prohibidas-2005.pdf > acceso 22/05/07

Espinosa-Chávez F., F. Martínez-Jerónimo \& R. Ramírez-Granados. 1992. Tasa de filtración y cultivo de Moina macrocopa (Crustacea: Cladocera) alimentada con Scenedesmus incrassatulus (Chlorophyceae) y estiércol vacuno digerido. Anales del Instituto de Ciencias del Mar y Limnologia. Pp. 9.
Instituto Nacional de Ecología. 2004. El lindano en México. Plan Nacional de Implementación de la Convención de Estocolmo sobre Contaminantes Orgánicos Persistentes y de los Planes de Acción Nacional sobre el Lindano, Dioxinas, Furanos y Hexaclorobenceno. Pp

Iannacone, J., Alvariño, L., Caballero C. y J. Sánchez. 2000. Cuatro Ensayos Ecotoxicológicos para Evaluar Lindano y Clorpirifos. Gayana 64(2): 139-146.

ISO. 1982. Water quality - Determination of the inhibition of the inmobilility of Daphnia magna Straus (Cladocera Crustacea). ISO 6341. Suiza. 11 pp.

Núñez, M. \& J. Hurtado. 2005. Bioensayos de toxicidad aguda utilizando Daphnia magna Straus (Cladocera, Daphniidae) desarrollada en medio de cultivo modificado. Rev. peru. biol. 12(1): 165-170.

Rico Martínez, R. 2000. Desarrollo de bioensayos toxicológico y su aplicación en programas de monitoreo de la calidad de pozos y tomas de agua del municipio de Aguascalientes. Consejo Nacional de Ciencias y Tecnología. Pp. 37.

Silva, J., G. Torrejón, E. Bay-Schith \& A. Larrain. 2003. Calibración del bioensayo de toxicidad aguda con Daphnia pulex (Crustacea: Cladocera) usando un tóxico de referencia. Gayana 67(1): 87-96.

Sosa, A., L. Di marco, R. Ferrari \& M. L. Mattassini. 1999. Plaguicidas y trabajo rural en la provincia de Tucumán, ¿un problema ambiental?. Revista Avances en Energías Renovables y Medio Ambiente 3: 9-12.

US EPA. 1988. Short -Term methods for estimating the chronic toxicity of effluents and receiving waters to marine and estuarine organisms. EPA/600/4-87/028. Pp. 398 - 416. 


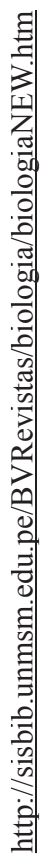

